\title{
Микроструктура и фазовый состав сплава дисилицидов железа и хрома
}

\author{
(С) Е.И. Суворова ${ }^{1}$, Ф.Ю. Соломкин ${ }^{2}$, Н.А. Архарова ${ }^{1}$, Н.В. Шаренкова ${ }^{2}$, Г.Н. Исаченко ${ }^{2}$ \\ ${ }^{1}$ Федеральный научно-исследовательский центр „Кристаллография и фротоника“ \\ Институт кристаллографиии им. А.В. Шубникова Российской академии наук, \\ 119333 Москва, Россия \\ ${ }^{2}$ Физико-технический институт им. А.Ф. Иоффе Российской академии наук, \\ 194021 Санкт-Петербург, Россия \\ E-mail: f.solomkin@mail.ioffe.ru
}

Поступила в Редакцию 20 октября 2021 г.

В окончательной редакции 25 октября 2021 г.

Принята к публикации 25 октября 2021 г.

\begin{abstract}
Методами сканирующей и просвечивающей электронной микроскопии, электронной дифракции и рентгеновской энергодисперсионной спектрометрии исследован фазовый состав, микроструктура и границы раздела фаз неупорядоченного сплава $\mathrm{CrSi}_{2}-\mathrm{FeSi}_{2}$, полученного при спонтанной кристаллизации (до и после отжига). Неотожженные образцы имели в своем составе фазы $\mathrm{CrSi}_{2}$ с гексагональной структурой $P_{4} 22$ и $\mathrm{FeSi}_{2}$ с тетрагональной структурой $P 4 / m m m$. В результате отжига произошло фазовое превращение тетрагонального $\mathrm{FeSi}_{2}$ в ромбическую модификацию Cmса. Внутри областей ромбического $\mathrm{FeSi}_{2}$ наблюдали выделения кубического моносилицида железа $\mathrm{FeSi}$ с пространственной группой $P 213$, нанопреципитатов кремния и силицида кремния $\mathrm{Cr}_{5} \mathrm{Si}_{3}$ с тетрагональной структурой $I 4 / \mathrm{mcm}$. Для фаз (ди)силицидов железа во всех образцах характерно наличие примесных межузельных атомов $\mathrm{Cr}$ до 2.0 ат\%. Структура фазы дисилицида хрома $\mathrm{CrSi}_{2}$ до и после отжига не изменяется, концентрация примесных атомов железа составляет $\sim 0.7$ ат\%. Установлены ориентационные соотношения между кристаллическими решетками фаз и вычислены деформации из-за несоответствия кристаллических решеток.
\end{abstract}

Ключевые слова: дисилицид хрома, дисилицид железа, граница раздела фаз, просвечивающая электронная микроскопия, рентгеновская энергодисперсионная спектрометрия.

DOI: $10.21883 /$ FTP.2022.02.51960.33

\section{1. Введение}

Термоэлектрики на базе силицидов $3 d$ переходных металлов представляют интерес для производства термоэлектрогенераторов и сенсорных устройств благодаря их химической и механической стойкости в окислительной среде при высоких температурах, их совместимости с кремниевой технологией, возможностью выбора среди них материалов $n$ - и $p$-типа проводимости. Особый интерес при создании термоэлектрических преобразователей представляет синтез анизотропных многофазных материалов. Границы раздела фаз являются полноправной третьей фазой со своими структурой и свойствами и, в зависимости от соотношения размерных параметров микроструктуры, могут играть решающую роль в физических свойствах.

В работе [1] была показана принципиальная возможность создания механически прочного материала на базе среднетемпературных термоэлектриков $\mathrm{CrSi}_{2}$ и $\mathrm{FeSi}_{2}$ с высокой анизотропией термоэлектрических параметров. При направленной кристаллизации наблюдалось образование слоистой микроструктуры с периодическим повторением слоев $\mathrm{CrSi}_{2}$ и $\mathrm{FeSi}_{2} . \mathrm{CrSi}_{2}$ - нетоксичный термоэлектрик с проводимостью $p$-типа и шириной запрещенной зоны 0.35 эВ обладает значительным фактором мощности с максимумом $45 \mathrm{M \kappa} \mathrm{BT} /\left(\mathrm{K}^{2} \cdot \mathrm{cm}\right)$ при
$T=600 \mathrm{~K}$, способен работать в агрессивных средах без специальной защиты. Текстурированный $\mathrm{CrSi}_{2}$ может использоваться для создания анизотропных термоэлектрических генераторов, работающих в широком интервале температур (200-1000 K) [2]. Материал имеет широкую область гомогенности, поэтому термоэлектрические свойства образцов сильно зависят от условий синтеза, и стехиометрии [3].

Дисилицид железа $\mathrm{FeSi}_{2}$, так же как и $\mathrm{CrSi}_{2}$, является нетоксичным термоэлектрическим материалом, способным работать в агрессивных средах без специальной защиты. $\mathrm{FeSi}_{2}$ образует кристаллы двух модификаций: высокотемпературной фазы $\alpha-\mathrm{FeSi}_{2}$ с тетрагональной структурой и металлическим типом проводимости, и низкотемпературной фазы $\beta-\mathrm{FeSi}_{2}$ с ромбической структурой - полупроводник с шириной запрещенной зоны 0.85 эВ [4]. При отжиге $\alpha-\mathrm{FeSi}_{2}$ при температуре, соответствующей твердотельному фазовому переходу $(1070-1170 \mathrm{~K})$, происходит полиморфное превращение в фазу $\beta-\mathrm{FeSi}_{2}$. Поэтапный отжиг $\alpha-\mathrm{FeSi}_{2}$ приводит к постепенному переходу от полуметалла к полупроводнику $\beta$-FeSi ${ }_{2}$, без механического разрушения образцов [5].

В случае длительной направленной кристаллизации (метод Бриджмена) в температурной зоне, соответствующей твердотельному фазовому переходу, происходит отжиг изначально кристаллизовавшейся высокотемпера- 


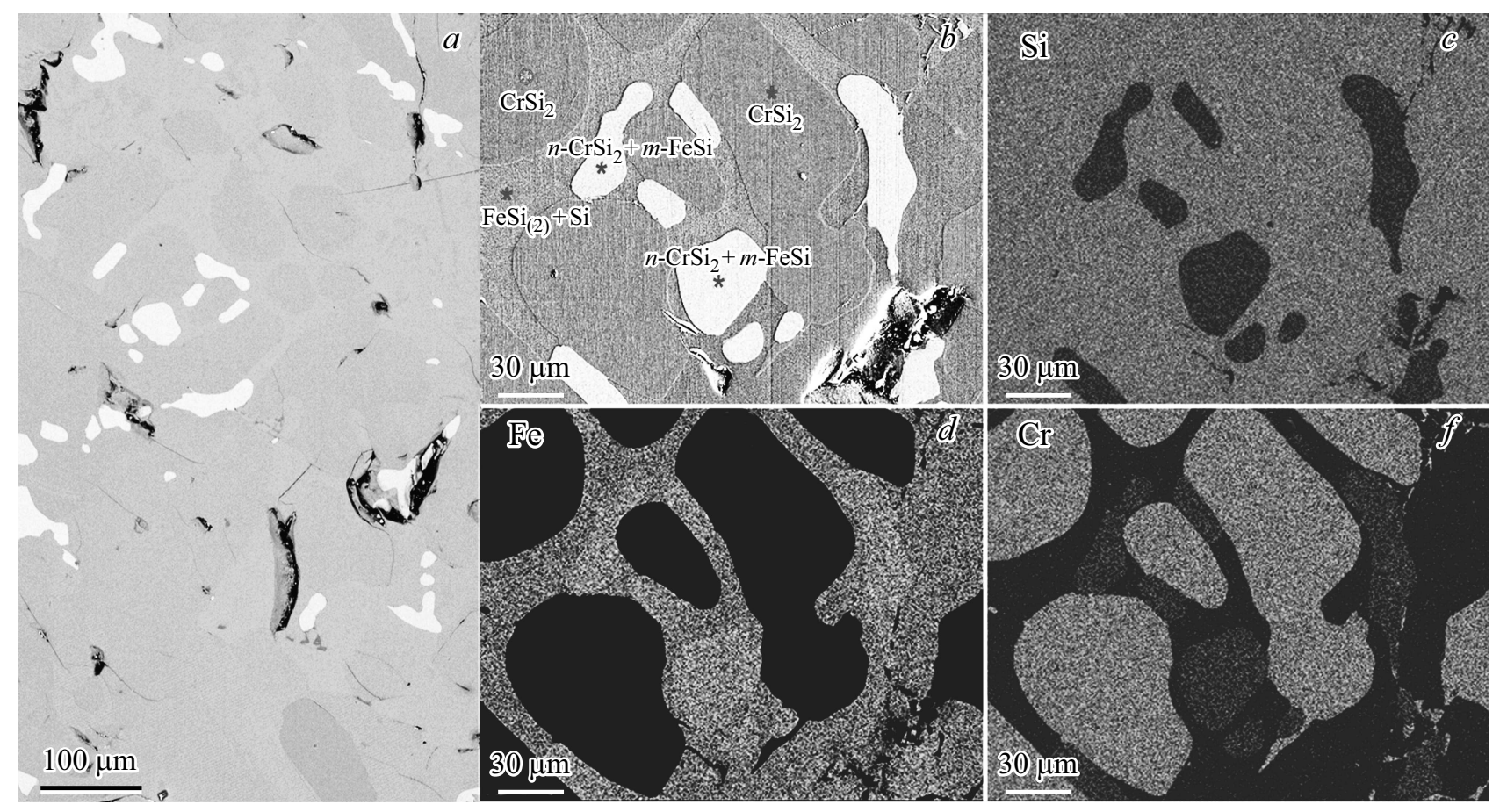

Рис. 1. СЭМ-изображение (обзорное) неупорядоченного сплава $\mathrm{CrSi}_{2}-\mathrm{FeSi}_{x}(a)$, распределение фаз $(b)$ и соответствующее распределение элементов $\mathrm{Si}, \mathrm{Fe}$ и $\mathrm{Cr}$ по данным ЭДС $(c, d, f)$.

турной фазы с превращением ее в $\beta$-FeSi 2 [6]. Известно, что при длительной направленной кристаллизации за счет разных коэффициентов диффузии компонентов расплава, находящегося в температурном градиенте, происходит их разгонка по объему слитка. Поэтому в данной работе для выяснения фазового состава, дефектных состояний и состояния границ раздела фаз после синтеза использовалась спонтанная кристаллизация.

\section{2. Материалы и методы исследования}

Синтез образцов проводился в вакууме, прямым сплавлением мелкодисперсных компонент $\mathrm{Cr}$, $\mathrm{Fe}$ и $\mathrm{Si}$, взятых в соотношении, соответствующем стехиометрии $\mathrm{CrFeSi}_{4}$, при $T=1723 \mathrm{~K}$. После спонтанной кристаллизации образцы отжигали на воздухе при $T=1073 \mathrm{~K}$ в течение 72 ч.

Методами сканирующей электронной микроскопии $(\mathrm{CЭM})$, просвечивающей электронной микроскопии (ПЭМ), электронной дифракции (ЭД) и рентгеновской энергодисперсионной спектрометрии (ЭДС) исследованы химический и фазовый состав образцов до и после отжига в тонких частицах, полученных скалыванием массивных образцов. СЭМ (FEI Scios с ускоряющими напряжениями в интервале 5-15 кэВ) использовалась для получения обзорных изображений с распределением зерен различных фаз. ПЭМ, ЭДС и электронографическое исследование на микро- и наноуровне проводились в микроскопе Tecnai Osiris с ускоряющим напряжением 200 кВ. Обработка и интерпретация полученных данных проводилась с помощью программ Gatan Digital Micrograph (GATAN), JEMS [7], ESPRIT (Bruker). СЭМ-изображения и ЭДС предоставляют возможность оценить размеры и форму зерен, распределение элементов и фаз в образцах (рис. 1).

\section{3. Результаты исследования микроструктуры}

Материал отожженных и неотожженных образцов состоит из смеси случайно распределенных зерен фаз силицидов $\mathrm{Cr}$ и $\mathrm{Fe}$. Светлые зерна на рис. 1 соответствуют фазам с большим средним атомным номером. Например, зерна (ди)силицида железа или комбинации силицида железа и примеси хрома будут всегда светлее по сравнению с зернами чистого $\mathrm{CrSi}_{2}$ (средний атомный номер 36) или комбинация $\mathrm{FeSi}_{2}$ и нановыделения $\mathrm{Si}$ (средний атомный номер 32).

Анализ ПЭМ-изображений и картин электронной дифракции показал, что $\mathrm{CrSi}_{2}$ с гексагональной структурой $P_{4} 22$ [8] образуется в синтезированных образцах до их отжига и не меняет своей структуры после отжига (рис. 2). Количественный ЭДС-анализ неотожженных образцов показал, что в зернах $\mathrm{CrSi}_{2}$ может содержаться не более $0.7 \mathrm{aT} \% \mathrm{Fe}$, а в решетке тетрагональной фазы $\alpha-\mathrm{FeSi}_{2}$ до $2 \mathrm{aT} \% \mathrm{Cr}$. Тетрагональный $\alpha-\mathrm{FeSi}_{2}$ со структурой $P 4 / \mathrm{mmm}[9]$ после отжига превращается в фазу $\beta-\mathrm{FeSi}_{2}$ с ромбической структурой $C m c a$ [10]. Кроме то- 

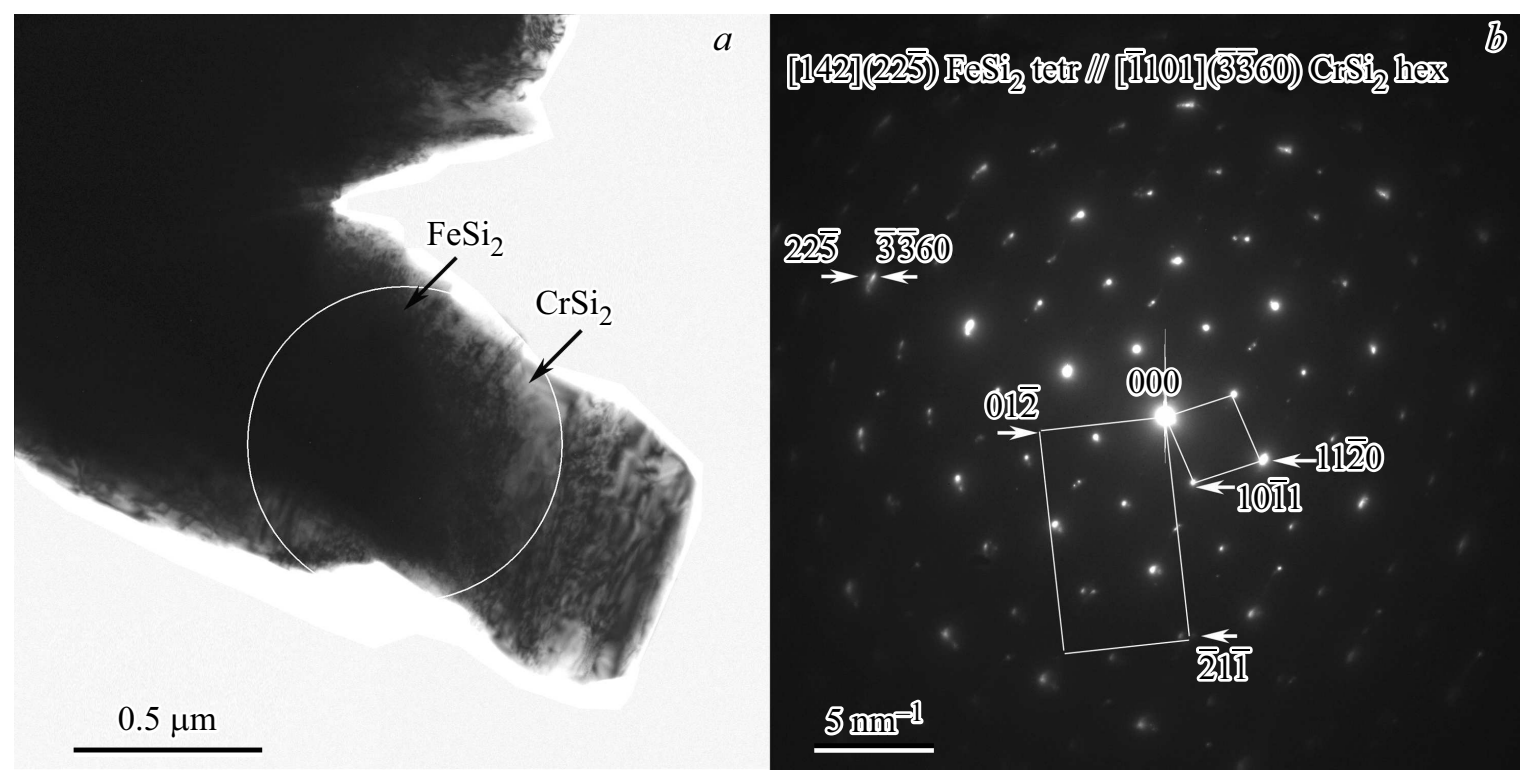

Рис. 2. ПЭМ-изображение $(a)$ и картина электронной дифракции $(b)$ с выделенными ячейками от $\mathrm{CrSi}_{2}$ и $\mathrm{FeSi}_{2}$ и ориентационными соотношениями.

го, внутри зерен $\beta$-FeSi 2 наблюдали выделение нанокристаллов $\mathrm{Cr}_{5} \mathrm{Si}_{3}$ с тетрагональной решеткой и вытянутых нанопреципитатов $\mathrm{Si}$, в то время как зерна $\mathrm{CrSi}_{2}$ не содержат примеси железа или выделений кремния. Были выявлены тонкие слои $\mathrm{FeSi}$ с кубической структурой P213 [11], граничащие с $\mathrm{CrSi}_{2}$. В таблице перечислены фазы силицидов в неупорядоченных сплавах $\mathrm{CrSi}_{2}-\mathrm{FeSi}_{2}$ до и после отжига.

Анализ картин электронной дифракции позволил определить несколько ориентационных соотношений для обоих типов образцов сплава. Обычной практикой является выражение ориентационных соотношений через плоскость и направление в одной фазе (преципитате), которые параллельны плоскости и направлению в другой фазе (матрице), для чего образец в электронном микроскопе наклоняется для получения дифракционной картины, в которой оси зоны (направления к плоскости картины) от обеих фаз совпадают. С помощью ориентационных соотношений можно оценить несоответствия между кристаллическими решетками и возможные деформации, а также степень когерентности межфазной границы.
На рис. 2 представлено ПЭМ-изображение от неотожженного образца (рис. 2,a) с соответствующей электронной дифракцией (рис. 2, $b$ ), на которой присутствуют рефлексы от гексагонального $\mathrm{CrSi}_{2}$ и тетрагонального $\mathrm{FeSi}_{2}$. Между двумя фазами на экспериментальных электронограммах были выявлены следующие ориентационные соотношения:

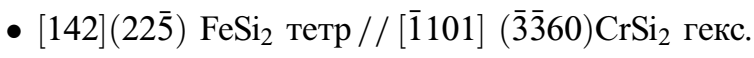

- $[881](1 \overline{1} 0) \mathrm{FeSi}_{2}$ тетр // [112̄0](22̄00) $\mathrm{CrSi}_{2}$ гекс.

Несоответствие $\delta$ между решетками, рассчитанное по формуле

$$
\delta=\frac{2\left(d_{\mathrm{Fe}}-d_{\mathrm{Cr}}\right)}{d_{\mathrm{Fe}}+d_{\mathrm{Cr}}},
$$

где $d_{\mathrm{Fe}}$ и $d_{\mathrm{Cr}}-$ межплоскостные расстояния, от соответствующих плоскостей двух фаз силицидов $\mathrm{Fe}$ и $\mathrm{Cr}$ $\left(d_{22-5} \mathrm{FeSi}_{2}\right.$ тетр $=0.0696 \mathrm{HM}, d_{-3-360} \mathrm{CrSi}_{2}$ гекс $=0.0738 \mathrm{HM}$, $d_{1-10} \mathrm{FeSi}_{2}$ тетр $=0.1906 \mathrm{HM}, d_{2-200} \mathrm{CrSi}_{2}$ гекс $\left.=0.1107 \mathrm{HM}\right)$ составляло $\sim 6 \%$ для первого соотношения и $0.5 \%-$ для второго.

Фазовый состав неупорядоченного сплава $\mathrm{CrSi}_{2}-\mathrm{FeSi}_{2}$ до и после отжига

\begin{tabular}{|c|c|c|c|c|}
\hline \multirow[b]{2}{*}{ Фазы } & \multicolumn{2}{|c|}{ До отжига } & \multicolumn{2}{|c|}{ После отжига } \\
\hline & Фаза & $\begin{array}{c}\text { Пространственная } \\
\text { группа }\end{array}$ & Фаза & $\begin{array}{c}\text { Пространственная } \\
\text { группа }\end{array}$ \\
\hline Силицид Fe & $\mathrm{FeSi}_{2}$ тетр & $P 4 / m m m$ & $\begin{array}{l}\mathrm{FeSi}_{2} \text { ромб } \\
\mathrm{FeSi} \text { куб }\end{array}$ & $\begin{array}{l}\text { Cmca } \\
\text { P213 }\end{array}$ \\
\hline $\begin{array}{l}\text { Силицид Cr } \\
\text { Кремний }\end{array}$ & $\begin{array}{l}\mathrm{CrSi}_{2} \text { гекс } \\
\text { Не выявлен }\end{array}$ & $\mathrm{P}_{4} 22$ & $\begin{array}{l}\mathrm{CrSi}_{2} \text { гекс } \\
\mathrm{Cr}_{5} \mathrm{Si}_{3} \text { тетр } \\
\mathrm{Si} \text { куб }\end{array}$ & $\begin{array}{c}\mathrm{Pb}_{4} 22 \\
\mathrm{I} 4 / \mathrm{mcm} \\
\mathrm{Fd}-3 \mathrm{~m}\end{array}$ \\
\hline
\end{tabular}



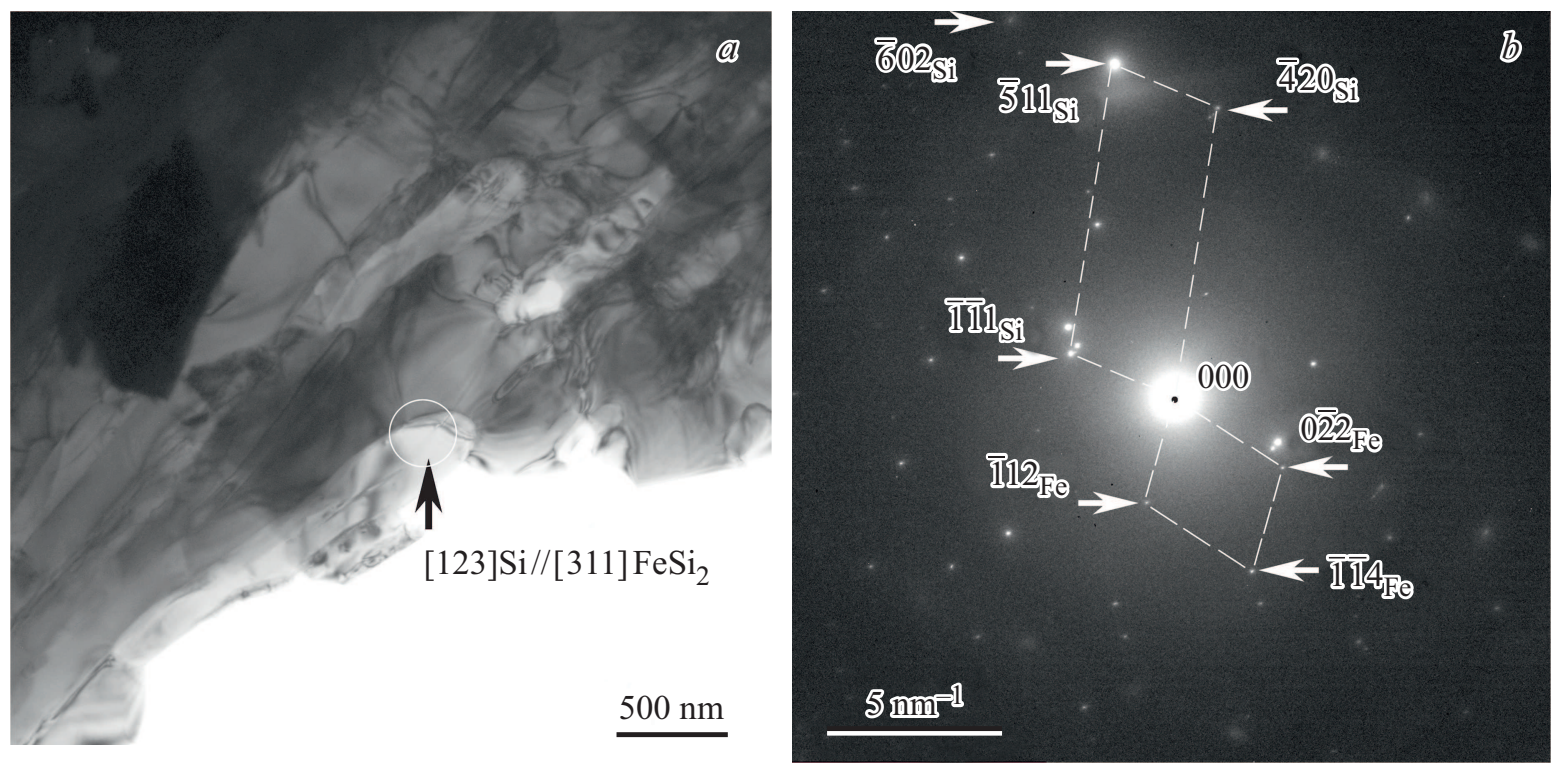

Рис. 3. ПЭМ-изображение зерна $\beta$ - $\mathrm{FeSi}_{2}$ с нановыделениями $\mathrm{Si}(a)$, картина электронной дифракции $(b)$ с рефлексами от $\beta$-FeSi 2 и $\mathrm{Si}$, полученная от области, выделенной кругом на ПЭМ-изображении.

Для отожженных образцов установлены следующие ориентационные соотношения и несоответствия $\delta \approx 1 \%$ между решетками $\mathrm{CrSi}_{2}$ гекс и $\mathrm{FeSi}_{2}$ ромб $\left(d_{6-33-3}\right.$ $\mathrm{CrSi}_{2}$ гекс $=0.0697, d_{0-88} \mathrm{FeSi}_{2}$ ромб $\left.=0.0691 \mathrm{HM}\right)$ :

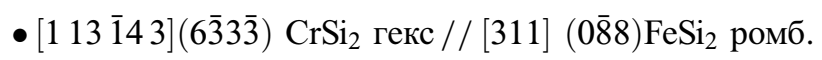

Как уже было сказано выше, в образцах после отжига наблюдали выделения кубического моносилицида железа $\mathrm{FeSi}$ в зернах гексагонального $\mathrm{CrSi}_{2}$ в виде тонких стержней шириной $\sim 50$ нм. Нанопреципитаты тетрагональной фазы $\mathrm{Cr}_{5} \mathrm{Si}_{3}$ [12] и кубического $\mathrm{Si}$ [13] (рис. 3) были выявлены в матричных зернах ромбического $\mathrm{FeSi}_{2}$. Ориентационные соотношения между матричными зернами $\mathrm{CrSi}_{2}$ и $\mathrm{FeSi}_{2}$ и нановыделениями вторичных фаз после отжига представлены далее:

- $[12 \overline{3} 0](0003) \mathrm{CrSi}_{2}$ гекс // [120](210)FeSi куб, $\delta \approx 8 \%$.

- $[112](\overline{1} \overline{5} 3) \mathrm{FeSi}_{2}$ ромб // [379](223) $\mathrm{Cr}_{5} \mathrm{Si}_{3}$ тетр,

$$
\delta \approx 0.4 \% \text {. }
$$

- $[311](\overline{2} \overline{2} 8) \mathrm{FeSi}_{2}$ ромб // [123](60) $) \mathrm{Si}$ куб, $\delta \approx 8 \%$.

Для определения несоответствия между кристаллическими решетками фаз взяты следующие межплоскостные расстояния: $d_{0003} \mathrm{CrSi}_{2 \text { гекс }}=0.2123 \mathrm{HM}$, $d_{-210} \mathrm{FeSi}_{\text {куб }}=0.2035 \mathrm{HM}, d_{-1-53} \mathrm{FeSi}_{2 \text { ромб }}=0.1326 \mathrm{HM}$, $d_{233} \mathrm{Cr}_{5} \mathrm{Si}_{3 \text { тетр }}=0.1321 \mathrm{HM}, d_{-2-28} \quad \mathrm{FeSi}_{2 \text { ромб }}=0.0933$, $d_{60-2} \mathrm{Si}_{\text {куб }}=0.0862$ нм. Таким образом, все экспериментально наблюдаемые ориентационные соотношения свидетельствуют об образовании полукогерентных межфазных границ, при этом релаксация напряжений происходит с помощью дислокаций несоответствия. Очевидно, что возможны и другие ориентационные соотношения в разных направлениях с другими численными несоответствиями.

Деформации несоответствия параметров решеток различных фаз в материалах, а также несоответствие их коэффициентов теплового расширения порождают механические напряжения, которые снимаются образованием дефектов, например, трещин и каверн, которые выявляются на полированной поверхности как отожженных, так и неотожженных образцов (рис. 1,a). Деформация, вызванная различиями при тепловом расширении, может быть представлена в виде

$$
\delta_{\text {therm }}=\frac{d_{1}^{R T}\left(\alpha_{1}-\alpha_{2}\right) \Delta T}{d_{2}^{R T}\left(1+\alpha_{2} \Delta T\right)},
$$

где $d_{1}^{R T}$ и $d_{2}^{R T}$ - соответствующие межплоскостные расстояния в решетках двух фаз при комнатной температуре, $\Delta T$ - разница между температурой синтеза и комнатной температурой, $\alpha_{1}$ и $\alpha_{2}-$ коэффициенты теплового расширения фаз двух соседних зерен.

Значения коэффициентов линейного термического расширения (средние с учетом анизотропии) для $\beta-\mathrm{FeSi}_{2}$ находятся в интервале $10.6-11.8 \cdot 10^{-6} \mathrm{~K}^{-1}$ [14], a для $\mathrm{CrSi}_{2}$ интервал значений составляет $10.6-14.2 \cdot 10^{-6} \mathrm{~K}^{-1} \quad[15] \quad$ при температурах от комнатной до $1173 \mathrm{~K}$. Вклад в появление деформаций решетки твердого раствора благодаря разности коэффициентов теплового расширения составляет $\sim 0.5 \%$, в то время как несоответствие параметров решетки $\mathrm{FeSi}_{2}$ и $\mathrm{CrSi}_{2}$ в неотожженных образцах доходит до $6 \%$, что является основной причиной напряжений, при релаксации которых образуются дефекты. После отжига твердого раствора происходит выделение еще нескольких фаз с 8-процентным несоответствием между 
решетками матричных зерен и преципитатов, что также может приводить к напряжениям и в конечном итоге к появлению трещин в образцах.

\section{4. Заключение}

1. Данные, полученные при исследовании микроструктуры и состава неупорядоченного сплава с изначально заложенной при синтезе стехиометрией $\mathrm{CrFeSi}_{4}$, свидетельствуют о стабильности фазы $\mathrm{CrSi}_{2}$, которая после отжига не содержит ни примесей железа, ни выделений кремния.

2. Тетрагональный $\alpha-\mathrm{FeSi}_{2}$ после отжига превращается в $\beta$-FeSi 2 фазу с ромбической структурой, в которой наблюдается присутствие нанопреципитатов силицида $\mathrm{Cr}_{5} \mathrm{Si}_{3}$ с тетрагональной решеткой и вытянутых преципитатов $\mathrm{Si}$, что в случае направленной кристаллизации сплава может быть технологическим фактором для управления физическими свойствами композита.

3. Между кристаллическими решетками фаз в различных кристаллографических направлениях установлены численные ориентационные несоответствия. Релаксация деформаций, вызванных несоответствиями между решетками, может проводить к образованию дислокаций на границах раздела с последующим растрескиванием материала.

\section{Финансирование работы}

Работа поддержана Министерством науки и высшего образования Российской Федерации.

\section{Конфликт интересов}

Авторы заявляют, что у них нет конфликта интересов.

\section{Список литературы}

[1] Ф.Ю. Соломкин, В.К. Зайцев, С.В. Новиков, А.Ю. Самунин, Д.А. Пшенай-Северин, Г.Н. Исаченко. ЖТФ, 84, 106 (2014).

[2] M.I. Fedorov, V.K. Zaitsev. Handbook of Thermoelectric, ed. by D.M. Rowe (N.Y., CRC press., 2006) p. 31-2.

[3] Ф.Ю. Соломкин, В.К. Зайцев, С.В. Новиков, Ю.А. Самунин, Г.Н. Исаченко. ЖТФ, 83 (2), 141 (2013).

[4] U. Birkholz, E. Gross, U. Stohrer. Handbook of Thermoelectrics, ed. by D.M. Rowe (N.Y., CRC Press, 1995) p. 287.

[5] Ф.Ю Соломкин, Д.А. Пшенай-Северин, А.Ю. Самунин, Г.Н. Исаченко. Тез. докл. Межсгос. конф. „Термоэлектрики и их применения - 2014“ (СПб., Россия, 2014) с. 407.

[6] Ф.Ю. Соломкин, А.Ю. Самунин, Н.Ф. Картенко, А.С. Колосова. IX Межсгос. сем. „Термоэлектрики и их применения - 2004“ (СПб., Россия, 2004) с. 260.
[7] P. Stadelmann. 2017. JEMS, описание программы можно найти на https://www.jems-swiss.ch/

[8] K.Tanaka, K. Nawata, M. Koiwa, M. Yamaguchi, H. Inui. Mater. Res. Soc. Symp. Proc., 646, N 4.3 .1 (2001).

[9] B. Aronsson. Acta Chem. Scand., 14, 1414 (1960).

[10] Y. Dusausoy, J. Protas. Acta Cryst. B, 27, 1209 (1971).

[11] L. Vocadlo, K.S. Knight, G.D. Price, I.G. Wood. Phys. Chem. Miner., 29, 132 (2002).

[12] C.H. Dauben, D.H. Templeton, C.E. Myers. J. Phys. Chem., 60, 443 (1956).

[13] W.L. Bond, W. Kaiser. J. Phys. Chem. Sol., 16, 44 (1960).

[14] M. Imai, Y. Isoda, H. Udono. Intermetallics, 67, 75 (2015).

[15] Б.С. Рабинович, И.З. Радовский, П.В. Гельд. Порошковая металлургия, 7, 879 (1968).

Редактор А.Н. Смирнов

\section{Microstructure and phase composition of an alloy of iron and chrome disilicides}

\author{
E.I. Suvorova ${ }^{1}$, F.Yu. Solomkin ${ }^{2}$, N.A.Arkharova ${ }^{1}$, \\ N.V. Sharenkova ${ }^{2}$, G.N. Isachenko ${ }^{2}$ \\ ${ }^{1}$ Shubnikov Institute of Crystallography, \\ Federal Scientific Research Centre \\ "Crystallography and Photonics", \\ Russian Academy of Sciences, \\ 19333 Moscow, Russia \\ ${ }^{2}$ loffe Institute, \\ 194021 St. Petersburg, Russia
}

Abstract The phase composition, microstructure, and interphase interfaces of the disordered $\mathrm{CrSi}_{2}-\mathrm{FeSi}_{2}$ solid solution obtained by spontaneous crystallization (before and after annealing) have been investigated by scanning, transmission electron microscopy, electron diffraction, and $X$-ray energy dispersive spectrometry. The as-grown samples contained the phases of $\mathrm{CrSi}_{2}$ with the $P 6_{4} 22$ hexagonal structure and $\mathrm{FeSi}_{2}$ with the $P 4 / m m m$ tetragonal structure. Annealing of the samples led to the phase transformation of tetragonal $\mathrm{FeSi}_{2}$ into the orthorhombic modification Cmca. Precipitates of cubic iron monosilicide $\mathrm{FeSi}$ with space group $P 213$, nano-precipitates of $\mathrm{Si}$ and silicon silicide $\mathrm{Cr}_{5} \mathrm{Si}_{3}$ with a tetragonal structure $14 / \mathrm{mcm}$ were observed inside the $\mathrm{FeSi}_{2}$ grains. Impurities of interstitial $\mathrm{Cr}$ atoms with a concentration up to 2.0 at $\%$ are found in iron (di)silicides grains in all samples. The structure of the $\mathrm{CrSi}_{2}$ phase remains unchanged after annealing; the concentration of impurity iron atoms is about $0.7 \mathrm{at} \%$. Orientation relationships between the crystal lattices of the phases are established and strains due to the mismatch of the crystal lattices are determined.

\section{Публикация материалов Конференция завершена.}

